ANNALES

POLONICI MATHEMATICI

XII (1962)

\title{
Sur la résolution des équations algébriques par la méthode de Euler
}

\author{
par H. Górecki et A. B. Turowicz (Kraków)
}

§ 1. Dans la présente note nous donnons les formules permettant de calculer au moyen de séries infinies les racines des équations algébriques dont toutes les racines sont réelles. Au $\$ 2$ nous traitons le cas d'une équation du troisième degré. La formule (4) est plus commode que la méthode trigonométrique usuelle. Le $\S 3$ contient des remarques complémentaires sur l'équation du troisième degré. Au $\$ 4$ nous établissons une formule approchée très simple pour la racine de l'équation cubique. Enfin le $\$ 5$ concerne les formules pour le calcul des racines des équations de degré $n>3$.

§ 2. Soit

$\left(a_{0}, a_{1}, a_{2}, a_{3}\right.$ réels). On suppose que le racines de $(1) x_{1}, x_{2}, x_{3}$ soient réelles et que $x_{1}<x_{2}<x_{3}$. Les racines de l'équation $f^{\prime}(x)=0$ sont désignées par $\alpha_{1}, a_{2}$, et la racine de $f^{\prime \prime}(x)=0$ par $x_{0}$. On posera $f\left(x_{0}\right)=y_{0}$, $f^{\prime}\left(x_{0}\right)=y_{0}^{\prime}, f^{\prime \prime \prime}\left(x_{0}\right)=y_{0}^{\prime \prime \prime}$. On vérifie aisément que

$$
y_{0}=f\left(x_{0}\right)=\frac{1}{2}\left[f\left(a_{1}\right)+f\left(\alpha_{2}\right)\right] \text {. }
$$

Soit

$$
q=\frac{y_{0}^{2} y_{0}^{\prime \prime \prime}}{y_{0}^{\prime 3}} .
$$

THÉORÈME 1. Si l'équation (1) a trois racines réelles distinctes, on a

$$
x_{2}=x_{0}-\frac{y_{0}}{y_{0}^{\prime}} \sum_{s=0}^{\infty}\left(\begin{array}{c}
3 s \\
s
\end{array}\right) \frac{1}{2 s+1}\left(-\frac{q}{6}\right)^{s} .
$$

Démonstration. La fonction $y=f(x)$ est monotone dans l'intervalle $\left(a_{1}, a_{2}\right)$. Désignons la fonction inverse par $x=g(y)$. Puisque $a_{1}<x_{0}$ $<a_{2}$, on peut développer $g(y)$ en série de Taylor autour du point $y_{0}$ :

$$
g(y)=g\left(y_{0}\right)+\sum_{n=1}^{\infty} \frac{\left(y-y_{0}\right)^{n}}{n !} g^{(n)}\left(y_{0}\right) .
$$

Annales Polonicl Mathematicl XII 
Pour déterminer le rayon de convergence de (5), nous envisageons cette série dans le plan de la variable complexe $y$. Les seuls points singuliers de $g(y)$ sont $y=f\left(a_{1}\right)$ et $y=f\left(a_{2}\right)$, où la dérivée $g^{\prime}(y)$ devient infinie. En vertu de (2) le rayon de convergence est égal à

$$
\left|y_{0}-f\left(a_{1}\right)\right|=\left|y_{0}-f\left(a_{2}\right)\right| \text {. }
$$

Par conséquent la série (5) est convergente pour tout $y \in\left(f\left(a_{1}\right), f\left(a_{2}\right)\right)$ ( $y$ réel), et puisque $f\left(a_{1}\right) \cdot f\left(a_{2}\right)<0$ on peut mettre $y=0$. Comme $g(0)=x_{2}$, $g\left(y_{0}\right)=x_{0}$, on a

$$
x_{2}=x_{0}+\sum_{n=1}^{\infty}(-1)^{n} \frac{y_{0}^{n}}{n !} g^{(n)}\left(y_{0}\right)
$$

Nous calculons la dérivée $g^{(n)}\left(y_{0}\right)$ d'après la formule:

$$
g^{(n)}\left(y_{0}\right)=\sum_{z_{n}} \frac{\left(2 n-2-i_{1}\right) !(-1)^{n-1+i_{1}}}{i_{2} ! i_{3} ! \ldots i_{n} !\left(y_{0}^{\prime}\right)^{2 n-1}} \cdot\left(\frac{y_{0}^{\prime}}{1 !}\right)^{i_{1}} \cdot\left(\frac{y_{0}^{\prime \prime}}{2 !}\right)^{i_{2}} \ldots\left(\frac{y_{0}^{(n)}}{n !}\right)^{i_{n}}
$$

où $y_{0}^{(j)}=f^{(j)}\left(x_{0}\right), j=1, \ldots, n$, et $z_{n}$ est l'ensemble des suites finies $\left(i_{1}, \ldots, i_{n}\right)$, $i_{k}$ entiers, non négatifs, qui satisfont aux conditions:

$$
\sum_{k=2}^{n}(k-1) i_{k}=n-1, \quad i_{1}=n-1-\sum_{k=2}^{n} i_{k}
$$

(cf. A. Turowicz [1]).

On tire de $(1) y_{0}^{(k)}=0$ pour $k \geqslant 4$. On a aussi $y_{0}^{\prime \prime}=f^{\prime \prime}\left(x_{0}\right)=0$. Par conséquent pour qu'un terme de la somme (7) soit différent de 0 , il faut que $i_{2}=i_{4}=i_{5}=\ldots=i_{n}=0$. Les conditions (8) se réduisent ainsi aux suivantes:

$$
2 i_{3}=n-1, \quad i_{1}=n-1-i_{3} .
$$

On a donc pour $n$ pair $g^{(n)}\left(y_{0}\right)=0$. Pour $n=2 s+1, s=0,1,2$ il vient $i_{1}=i_{3}=s$.

$$
g^{(2 s+1)}\left(y_{0}\right)=\frac{(3 s) !(-1)^{s}}{8 !\left(y_{0}^{\prime}\right)^{4 s+1}} \cdot\left(y_{0}^{\prime}\right)^{s} \cdot\left(\frac{y_{0}^{\prime \prime \prime}}{6}\right)^{s}=\frac{(3 s) !}{s ! y_{0}^{\prime}}\left(-\frac{y_{0}^{\prime \prime \prime}}{6 y_{0}^{\prime 3}}\right)^{s}
$$

En substituant les expressions obtenues pour $g^{(n)}\left(y_{0}\right)$ dans (6) on obtient facilement (4).

\$ 3. En appliquant le critère de d'Alembert on trouve que la série (4) converge pour

$$
|q|<\frac{8}{9}
$$

Dans l'hypothèse que les trois racines de (1) soient réelles et distinctes en peut directement vérifier que la condition (11) est satisfaite (cf. la for- 
mule (18) ci-dessous). S'il y a une racine double, on a $|q|=\frac{8}{9}$, la série (4) converge encore, ce qu'on voit en appliquant le critère de Raabe, mais la formule (4) devient alors inutile.

Si l'équation (1) a deux racines complexes, la condition (11) n'est pas toujours satisfaite. Cependant, si elle subsiste, la formule (4) permet de calculer la racine réelle.

La rapidité de la convergence de la série (4) dépend de la position des racines $x_{1}, x_{2}, x_{3}$ : le quotient $q$ tend vers 0 , lorsque la racine $x_{2}$ converge vers le milieu du segment $\left(x_{1}, x_{3}\right)$.

§ 4. Une formule d'approximation. La série (4) donne le développement:

$$
\begin{aligned}
x_{2} & =x_{0}-\frac{y_{0}}{y_{0}^{\prime}}\left[1-\frac{1}{6}\left(q-\frac{1}{2} q^{2}+\frac{1}{3} q^{3}-\frac{55}{216} q^{4}+\frac{91}{432} q^{5}-\ldots\right)\right] \\
& =x_{0}-\frac{y_{0}}{y_{0}^{\prime}}\left[1-\frac{1}{6} h(q)\right] .
\end{aligned}
$$

Comparons la fonction $h(q)$ à la série:

$$
\log (1+q)=q-\frac{1}{2} q^{2}+\frac{1}{3} q^{3}-\frac{1}{4} q^{4}+\frac{1}{5} q^{5}-\ldots
$$

On voit que les trois premiers termes des développements de $h(q)$ et $\log (1+q)$ sont identiques et que les coefficients des quelques termes suivants diffèrent peu. On obtient donc la formule d'approximation:

$$
x_{2} \approx x_{0}-\frac{y_{0}}{y_{0}^{\prime}}\left[1-\frac{1}{6} \log (1+q)\right] \text {. }
$$

THÉonÈme 2. Si l'équation (1) a trois racines réelles et distinctes, on a

$$
x_{2}=x_{0}-\frac{y_{0}}{y_{0}^{\prime}}\left[1-\frac{1}{6} \log (1+q)\right]+\frac{2\left(x_{3}-x_{1}\right)}{9} \theta \vartheta(q)
$$

où $|\theta|<1$, et

$$
\vartheta(q)=\sum_{s=4}^{\infty}\left[\left(\begin{array}{c}
3 s \\
8
\end{array}\right) \frac{1}{2 s+1} \cdot \frac{1}{6^{8}}-\frac{1}{68}\right](-q)^{s}
$$

Démonstration. On a

$$
x_{2}-x_{0}+\frac{y_{0}}{y_{0}^{\prime}}\left[1-\frac{1}{6} \log (1+q)\right]=-\frac{y_{0}}{y_{0}^{\prime}} \sum_{s=4}^{\infty}\left[\left(\begin{array}{c}
3 s \\
s
\end{array}\right) \frac{1}{2 s+1} \cdot \frac{1}{6^{s}}-\frac{1}{6 s}\right](-q)^{s}
$$

Puisque $f^{\prime \prime}\left(x_{0}\right)=0$ on obtient

$$
\begin{gathered}
x_{0}=\frac{1}{3}\left(x_{1}+x_{2}+x_{9}\right), \quad y_{0}=a_{0}\left(x_{0}-x_{1}\right)\left(x_{0}-x_{2}\right)\left(x_{0}-x_{3}\right), \\
y_{0}^{\prime}=a_{0}\left[\left(x_{0}-x_{1}\right)\left(x_{0}-x_{2}\right)+\left(x_{0}-x_{1}\right)\left(x_{0}-x_{3}\right)+\left(x_{0}-x_{2}\right)\left(x_{0}-x_{3}\right)\right] .
\end{gathered}
$$


Soit $b=\frac{1}{2}\left(x_{3}-x_{1}\right), t=\frac{2 x_{2}-x_{1}-x_{3}}{x_{3}-x_{1}}$, donc

$$
x_{3}=x_{1}+2 b, \quad x_{2}=x_{1}+b+b t, \quad x_{0}=x_{1}+b+\frac{1}{3} b t .
$$

Un calcul facile donne:

done

$$
y_{0}=\frac{2 b^{3} a_{0}}{27}\left(9 t-t^{3}\right), \quad y_{0}^{\prime}=-\frac{b^{2}}{3} a_{0}\left(t^{2}+3\right)
$$

$$
-\frac{y_{0}}{y_{0}^{\prime}}=\frac{2 b}{9} \cdot \frac{9 t-t^{3}}{t^{2}+3}=\frac{x_{3}-x_{1}}{9} \cdot \frac{9 t-t^{3}}{t^{2}+3}
$$

On tire de la condition $\dot{x}_{1}<x_{2}<x_{3}$ l'inégalité: $-1<t<1$. Il suit de là que $-2<\frac{9 t-t^{3}}{t^{2}+3}<2$, donc

$$
\frac{9 t-t^{3}}{t^{2}+3}=2 \theta, \quad|\theta|<1
$$

Les relations (14), (15), (16) et (17) achèvent la démonstration.

La relation (13) ne permet pas d'évaleur l'erreur de l'approxima. tion (12), la différence $x_{3}-x_{1}$ n'étant pas connue. Cette formule est cependant suffisamment exacte pour les calculs techniques, parce que les valeurs de la fonction $\vartheta(q)$ sont très petites, comme le montre le tableau suivant. Pour calculer les valeurs de $\vartheta(q)$ du tableau nous exprimons $q$ par $t$ :

$$
q=-\frac{8}{9} \cdot \frac{\left(9 t-t^{3}\right)^{2}}{\left(t^{2}+3\right)^{3}}
$$

\begin{tabular}{|c|c|c|c|c|c|c|c|c|c|c|c|}
\hline$t$ & 0 & $\pm 0,1$ & $\pm 0,2$ & $\pm 0,3$ & $\pm 0,4$ & $\pm 0,5$ & $\pm 0,6$ & $\pm 0,7$ & $\pm 0,8$ & $\pm 0,9$ & \pm 1 \\
\hline$-q$ & 0 & 0,026343 & 0,101606 & 0,215265 & 0,352207 & 0,495644 & 0,629729 & 0,742033 & 0,82442 & 0,87328 & $\frac{8}{8}$ \\
\hline$\theta(q)$ & 0 & $2,25 \cdot 10^{-}$ & $.05 \cdot 10^{-}$ & $2,51 \cdot 10$ & $3,42 \cdot 10^{-5}$ & $2,06 \cdot 10^{-6}$ & $1,08 \cdot 10^{-}$ & $4,50^{\circ} \cdot 10^{-}$ & $1,63 \cdot 10^{-1}$ & $5,13 \cdot 10^{-}$ & $1,34 \cdot 1$ \\
\hline
\end{tabular}

et nous obtenons de (15):

$$
\vartheta(q)=\frac{4 t^{2}}{9-t^{2}}+\frac{1}{6} \log (1+q)
$$

Remarque 1. La formule (18) montre que toujours $q \leqslant 0$.

Remarque 2. On déduit de (15):

$$
x_{2}=x_{0}-\frac{y_{0}}{y_{0}^{\prime}}\left[1-\frac{1}{6} \log (1+q)\right]-\frac{y_{0}}{y_{0}^{\prime}} \vartheta(q) \text {. }
$$

Cette formule permet d'évaluer la marge de l'erreur de l'approximation (12) à l'aide du tableau de la fonction $\vartheta(q)$. 
§ 5. La méthode peut - avec une certaine restriction - être appliquée aux équations de degré $n>3$ dont toutes les racines sont réelles. Elle est basée sur le développement en série de Taylor de la fonction inverse de la fonction:

$$
y=f(x)=a_{0} x^{n}+a_{1} x^{n-1}+\ldots+a_{n}
$$

autour du point $y_{0}=f\left(x_{0}\right)$, où $f^{\prime \prime}\left(x_{0}\right)=0$; alors la formule (7), et par conséquent tous les calculs deviennent plus simples.

Désignons par $\alpha_{1}, \alpha_{2}$ deux racines consécutives de l'équation $f^{\prime}(x)=0$, par $x_{0}$ la racine de $f^{\prime \prime}\left(x_{0}\right)=0$ et par $\bar{x}$ la racine de $f(x)=0$, situées toutes les deux dans l'intervalle $\left(a_{1}, a_{2}\right)$. Alors:

TнÉoвغ̀me 3. Si l'équation $f(x)=0$ a $n$ racines réelles et si la série (22) est convergente, on $a$ :

$$
\bar{x}=x_{0}-\frac{y_{0}}{y_{0}^{\prime}}\left(1+\sum_{r=9}^{\infty} \sum_{W_{r}} \frac{\left(\sum_{k=9}^{n} k i_{k}\right) !}{\prod_{k=3}^{n} i_{k} !\left(\sum_{k=3}^{n}(k-1) i_{k}+1\right) !} \prod_{k=9}^{n}\left((-1)^{k} \frac{y_{0}^{k-1} \cdot y_{0}^{(k)}}{k ! y_{0}^{\prime k}}\right)^{i_{k}}\right.
$$

où $W_{r}$ est l'ensemble des suites finies $\left(i_{3}, i_{4}, \ldots, i_{n}\right), i_{3}, i_{4}, \ldots, i_{n}$ étant des nombres entiers, non négatifs, tels que

$$
\sum_{k=9}^{n}(k-1) i_{k}=r-1,
$$

et $y_{0}^{(k)}=f_{\left(x_{0}\right)}^{(k)}, y_{0}=f\left(x_{0}\right)$.

La démonstration est basée sur les relations (6), (7), (8), et sur l'égalité $y_{0}^{\prime \prime}=0$, et ne demande que de simples calculs. La convergence de (22) ne put être démontrée, parce que pour $n>3$ on a en général $f\left(x_{0}\right)$ $\neq \frac{1}{2}\left[f\left(a_{1}\right)+f\left(a_{2}\right)\right]$. C'est pourquoi cette convergence a été admise dans le théorème 3 comme hypothèse.

On a par exemple pour $n=4$ :

$$
\bar{x}=x_{0}-\frac{y_{0}}{y_{0}^{\prime}}\left[1+\sum_{n=3}^{\infty} \sum_{\nabla_{n}}\left(\begin{array}{c}
3 r+4 s \\
r+8
\end{array}\right)\left(\begin{array}{c}
r+s \\
r
\end{array}\right) \frac{1}{2 r+3 s+1}\left(\frac{-y_{0}^{2} y_{0}^{\mathrm{III}}}{6 y_{v}^{\prime 3}}\right)^{r}\left(\frac{y_{0}^{8} y_{0}^{\mathrm{IV}}}{24 y_{0}^{\prime 4}}\right)^{s}\right]
$$

où $V_{n}$ est l'ensemble des couples $(r, s), r, s$ entiers, non négatifs, $2 r+3 s$ $=n-1$.

Le théorème 3 permet de calculer les $(n-2)$ racines de $f(x)=0$ si toutes les racines de $f^{\prime \prime}(x)=0$ sont connues - pourvu que les séries (22) respectives soient convergentes.

On calcule ensuite facilement les deux racines de $f(x)=0$ restantes. De cette manière on pourrait résoudre une équation algébrique de degré 
arbitraire dont toutes les racines sont réelles. Dans le cas où $n$ eṣt un nombre pair, il faudrait calculer successivement les racines des équations

$$
f^{(n-2)}(x)=0, \quad f^{(n-4)}(x)=0, \quad \ldots, \quad f^{\prime \prime}(x)=0 .
$$

Dans le cas où $n$ est un nombre impair on prend les équations

$$
f^{(n-1)}(x)=0, \quad f^{(n-3)}(x)=0, \quad \ldots, \quad f^{\prime \prime}(x)=0 .
$$

Les calculs sont toutefois assez laborieux et il est à craindre qu'une des séries (22) soit divergente.

\section{Travaux cités}

[1] A. Turowicz, Sur les dérivées d'ordre supérieur d'une fonction inverse, Colloq. Math. 7 (1959), p. 83-87.

Reģu par la Rédaction le 9. 3. 1961 